\title{
Dual graft living donor liver transplantation - a case report
}

\author{
Nikam Vinayak* (D), Mohanka Ravi, Golhar Ankush, Bhade Rashmi, Rao Prashantha, Gadre Parul and Shrimal Anurag
}

\begin{abstract}
Background: Living donor liver transplantation (LDLT) has emerged as an equally viable option to deceased donor liver transplant for treating end stage liver disease patients. Optimising the recipient outcome without compromising donor safety is the primary goal of LDLT. Achieving the adequate graft to recipient weight ratio (GRWR) is important to prevent small for size syndrome which is an uncommon but potentially lethal complication of LDLT.

Case presentation: Here we describe a case of successful dual lobe liver transplant for a 32 years old patient with ethanol related end stage liver disease. A right lobe graft without middle hepatic vein and another left lateral sector graft were transplanted successfully. Recipient and both donors recovered uneventfully.
\end{abstract}

Conclusion: Dual lobe liver transplant is a feasible strategy to achieve adequate GRWR without compromising donor safety.

Keywords: Living donor liver transplantation, Deceased donor liver transplantation, End stage liver disease, Graft to recipient weight, Small-for-size syndrome, Future liver remnant

\section{Background}

Liver transplantation is a gold standard treatment for a patient with end-stage liver disease.

Living donor liver transplantation (LDLT) rate is increasing due to the shortage of deceased liver donations. Small for size syndrome and small donor liver remnant are two uncommon but potentially lethal complications of LDLT [1].

Small for size syndrome (SFSS) generally occurs when the graft recipient weight ratio (GRWR) is less than 0.8 . There is disequilibrium between increased metabolic demand and liver regeneration which leads to a severe graft dysfunction [2]. The clinical presentation of SFSS is intractable ascites, coagulopathy, and jaundice [2, 3]. The most accepted pathophysiological mechanism for SFSS is over-perfusion of transplanted liver graft [3].

LDLT is the most common type of liver transplant in India. Almost $33 \%$ of potential live donors are rejected as liver donors for adult recipients because of calculations suggesting a small for size (SFS) graft, a small future liver remnant (FLR) and steatosis [4].

\footnotetext{
* Correspondence: vinayak_nikam@yahoo.com

Institute of Liver Diseases, HPB Surgery and Transplantation, Global Hospital, 35, Dr. E Borges Road Opp. Shirodkar High School, Parel, Room No- 202, 2nd Floor, Mumbai, Maharashtra 400012, India
}

In recent years the subset of morbidly obese patients undergoing liver transplant is raising due to the increase in the incidence of non-alcoholic steatohepatitis (NASH) related end stage liver disease (ESLD). In order to overcome the problems of SFSS, optimise donor safety and increase the donor pool, dual graft liver transplant is the best feasible option. The first successful dual lobe LDLT using left lobe graft and left lateral section was performed by Lee et al. in year 2000 [5]. In this paper we are presenting a case of ethanol induced ESLD patient who successfully underwent dual graft LDLT by using right lobe and left lateral segment (LLS) grafts.

\section{Case presentation \\ Recipient details}

The recipient was a 32 years old male (weight- $90 \mathrm{~kg}$, height $-165 \mathrm{~cm}$, BMI- $32 \mathrm{~kg} / \mathrm{m}^{2}$ ) diagnosed with ethanol related ESLD, decompensated with jaundice, ascites, and hydrothorax (MELD Score- 34, CTP Score-11/C) (Table 1). He was evaluated as per our standard recipient evaluation protocol and placed on the deceased donor waiting list. Liver anatomy was evaluated by computed tomography (CT) triple phase abdomen which showed features of chronic liver disease with portal hypertension with a patent portal vein without any space

(c) The Author(s). 2019 Open Access This article is distributed under the terms of the Creative Commons Attribution 4.0 International License (http://creativecommons.org/licenses/by/4.0/), which permits unrestricted use, distribution, and 
Table 1 Clinical Data of Recipient

\begin{tabular}{ll}
\hline Age & 32 years \\
\hline Gender & Male \\
Blood group & O positive \\
Weight & $90 \mathrm{~kg}$ \\
Height & $165 \mathrm{~cm}$ \\
BMl & $32 \mathrm{~kg} / \mathrm{m}^{2}$ \\
CTP Score & $11 / \mathrm{C}$ \\
MELD & 34 \\
Alfa fetoprotein (AFP) & $4.3 \mathrm{ng} / \mathrm{ml}$ \\
\hline
\end{tabular}

occupying lesion in the liver. The patient was counselled for LDLT in view of a long waiting list for deceased donor liver transplant (DDLT). The patient needed dual lobe living donor liver graft due to inadequate partial liver graft volumes of individual donors.

\section{Donor details}

Donors were found to be fit for donor hepatectomy after clinical examination and laboratory tests (Table 2).

\section{Donor liver Volumetry and anatomical details \\ Donor I}

- Hepatic Artery: Hepatic artery proper was dividing into the left hepatic artery (LHA) and right hepatic artery (RHA). RHA had an extra-hepatic length of $27 \mathrm{~mm}$. Segment 4 artery was arising from LHA and there were no other accessory arteries identified (Fig. 1).

- Portal Vein: Portal vein anatomy was Nakamura Type A (Fig. 2). The length of right portal vein was $11 \mathrm{~mm}$. The significant crossover of the portal drainage was absent.

- Hepatic Veins: Two inferior right hepatic veins measuring $4.1 \mathrm{~mm}$ and $4.5 \mathrm{~mm}$ in diameter were noted draining into inferior vena cava. Significant

Table 2 Donor Details

\begin{tabular}{lll}
\hline Details & Donor I & Donor II \\
\hline Graft Type & Rt Lobe without & Lt Lateral Graft \\
& MHV & \\
Relation & Sister & Uncle \\
Age & 29 & 49 \\
Gender & Female & Male \\
Blood Group & O positive & O positive \\
BMI (kg/m ${ }^{2}$ ) & 22.9 & 23.4 \\
CT LAl & +11 & +7.8 \\
MR Elastography (mean & 2.2 kPa, 2-4\% & 2.4 kPa, 2-4\% \\
shear stiffness and Fat Fraction) & & Huang $\mathrm{A}_{1} \mathrm{~B}_{1}$ \\
MRCP- Bile Duct classification & Huang $\mathrm{A}_{4} \mathrm{~B}_{1}$ & \\
\hline
\end{tabular}

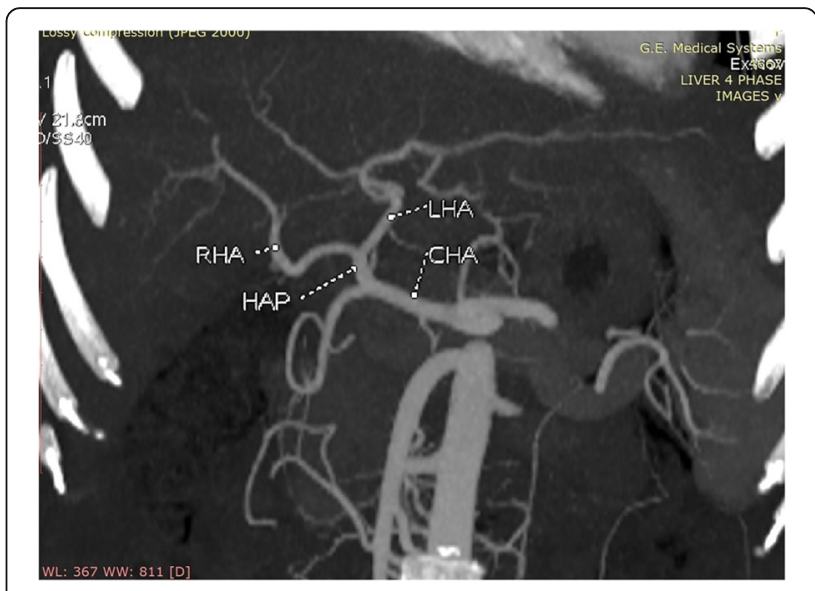

Fig. 1 Donor I - Hepatic Arterial Anatomy

size segment V (3.4 $\mathrm{mm})$ and VIII $(2.8 \mathrm{~mm})$ veins were draining into MHV (Figs. 3 and 4).

- Bile Duct - Bile duct anatomy was Huang Type $\mathrm{A}_{4} \mathrm{~B}_{1}$ (Fig. 5).

\section{Donor II}

- Hepatic artery: Hepatic artery proper (HAP) was dividing into LHA and RHA. LHA was arising from HAP immediately at its origin and supplied segments 2 and 3. Segment 4 artery was arising from RHA (Fig. 6).

- Portal Vein: Portal vein anatomy was Nakamura Type A (Fig. 7). Segment 4 portal vein (measuring $4.4 \mathrm{~mm}$ ) was arising from the left portal vein. No significant crossover of the drainage was noted.

- Hepatic veins: There was a short common channel between left hepatic vein (LHV) and Middle hepatic vein (MHV) measuring $5.8 \mathrm{~mm}$ in length (Figs. 8

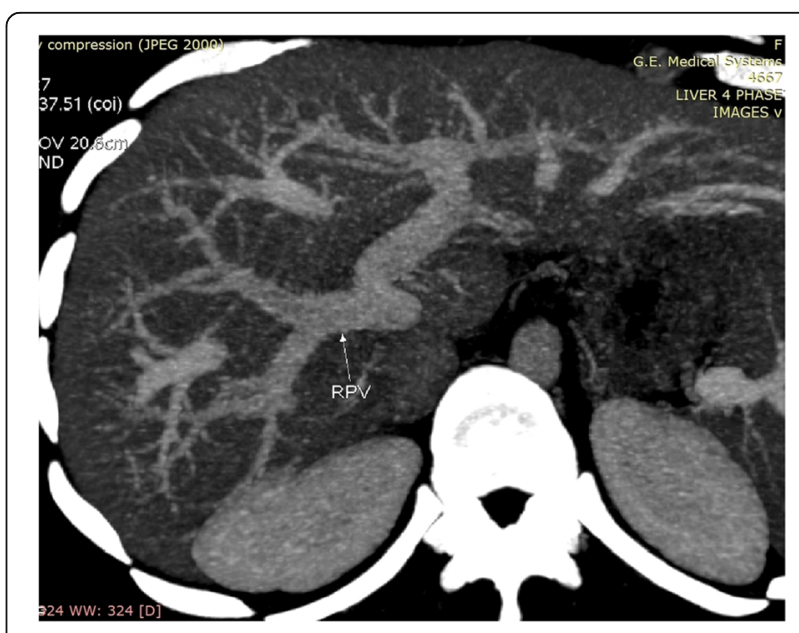

Fig. 2 Donor I - Portal Vein Anatomy 


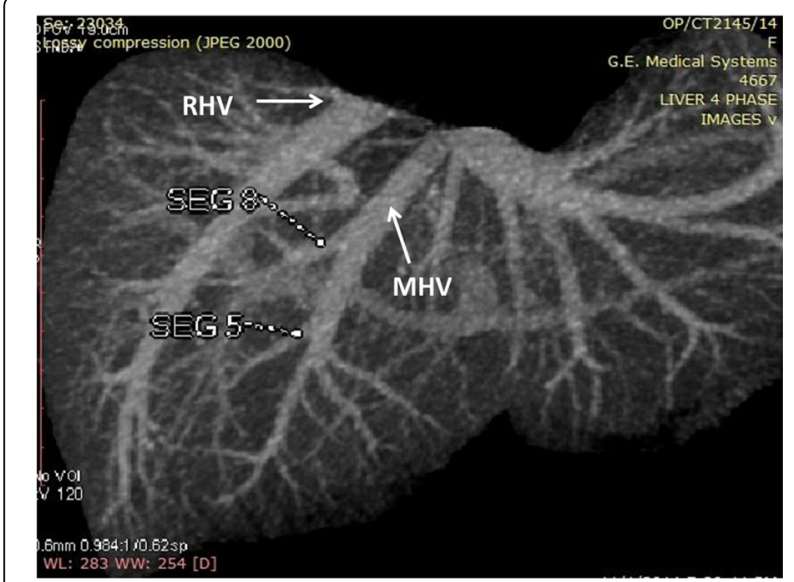

Fig. 3 Donor I - Hepatic Venous Anatomy

and 9). Left hepatic vein measured $6.5 \mathrm{~mm}$ near its ostium. A segment II/III vein measuring $9 \mathrm{~mm}$ in diameter was joined by segment III vein measuring $4.5 \mathrm{~mm}$ in diameter to form LHV. Another segment III vein (3 $\mathrm{mm}$ diameter) joined the LHV.

- Bile Duct - Bile duct anatomy was Huang Type $A_{1} B_{1}$ (Fig. 10).

CT Volumetric analysis of either donor livers showed a relatively small right liver lobes and calculated graft recipient weight ratios (GRWR) were 0.63 and 0.62 respectively. As neither of the two probable donors were suitable as a single donor (Table 3), the decision was made to perform LDLT using dual liver grafts. In this case, we chose the right liver lobe of Donor I together with the left lateral Sector of Donor II to get an adequate

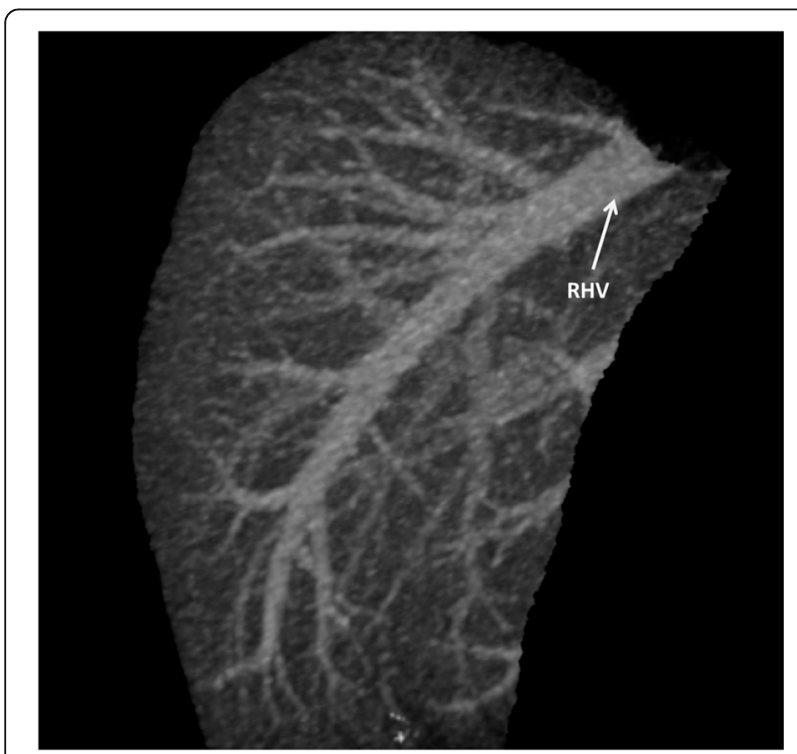

Fig. 4 Donor I Planned Right Lobe Without MHV Graft

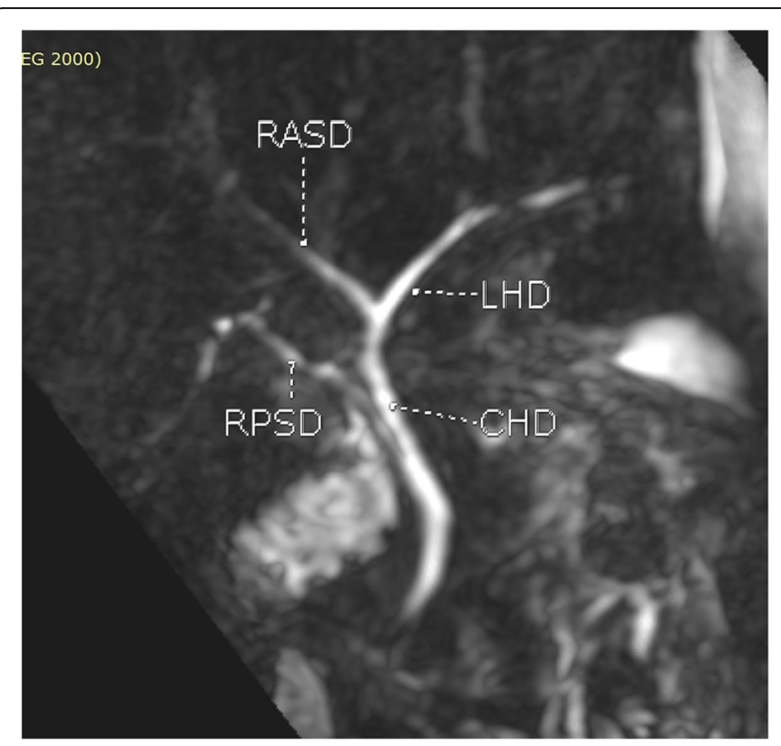

Fig. 5 Donor I- Bile Duct Anatomy (Huang Type A4B1)

GRWR. Future liver remnants (FLR) were adequate in both donors (donor I - 39\% and Donor II-70\%).

\section{Surgical procedure}

Risks and benefits of the surgery were discussed and informed consents were obtained from both donors and recipient. Recipient and both donor surgeries were started simultaneously.

\section{Recipient hepatectomy}

Surgical access was through a right sub-costal incision extended vertically in the midline till the xiphoid process. Recipient's cirrhotic liver was explanted as per standard hepatectomy procedure. Recipient received a $10 \mathrm{mg} / \mathrm{kg}$ dose of injection methylprednisolone immediately after liver explantation.

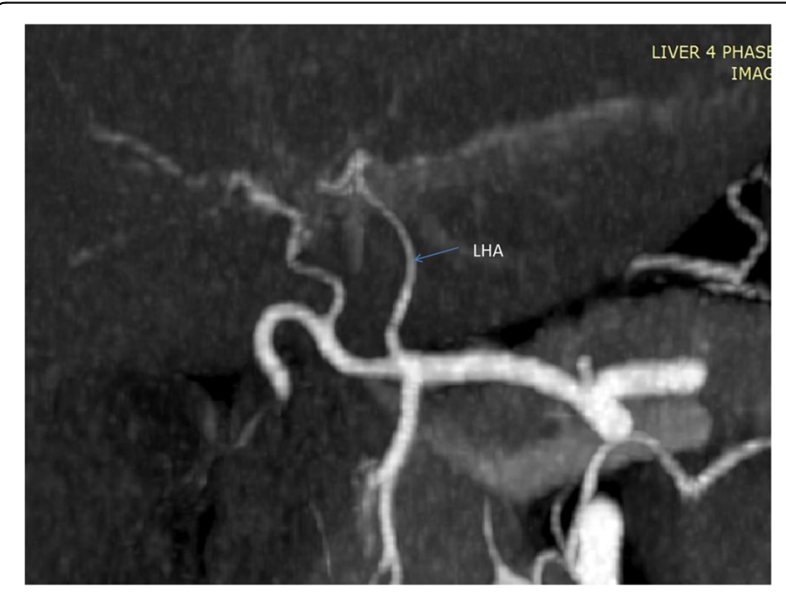

Fig. 6 Donor II - Hepatic Artery Anatomy 


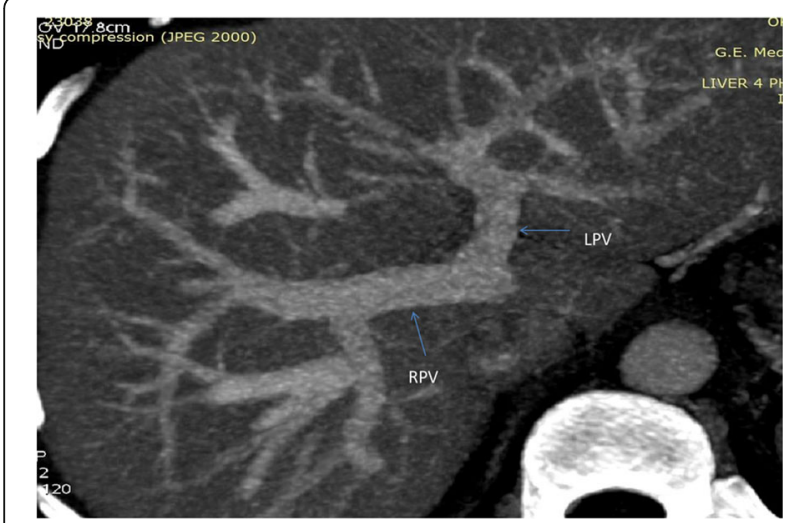

Fig. 7 Donor II - Portal Vein Anatomy

\section{Donor graft harvesting}

Right lobe without MHV graft was harvested from Donor I after the division of right anterior and posterior sectoral ducts (RASD and RPSD), right hepatic artery (RHA), right portal vein (RPV), right hepatic vein (RHV), and right inferior hepatic vein (RIHV). Left lateral lobe graft was harvested from Donor II after the division of the left hepatic duct, left hepatic artery (LHA), left portal vein (LPV) and LHV.

Actual harvested graft weights were $503 \mathrm{~g}$ (right lobe without MHV graft) and $374 \mathrm{~g}$ (Left lateral sector graft) (Fig. 11) giving GRWRs of 0.55 and 0.41 respectively (Total GRWR 0.96) (Table 4).

Operative time for Donor I and Donor II hepatectomy was $360 \mathrm{~min}$ and $310 \mathrm{~min}$ respectively with a blood loss of $450 \mathrm{ml}$ and $370 \mathrm{ml}$.

\section{Back table preparation}

Grafts were perfused with UW solution. As hepatic vein tributaries of segment $\mathrm{V}$ and VIII were less than $3.5 \mathrm{~mm}$ diameter, venous drainage of these segments

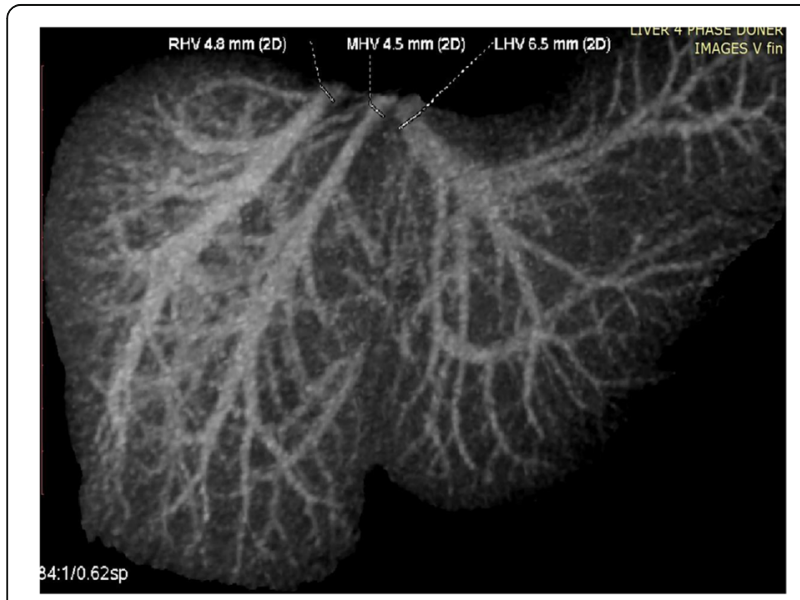

Fig. 8 Donor II - Hepatic Venous Anatomy

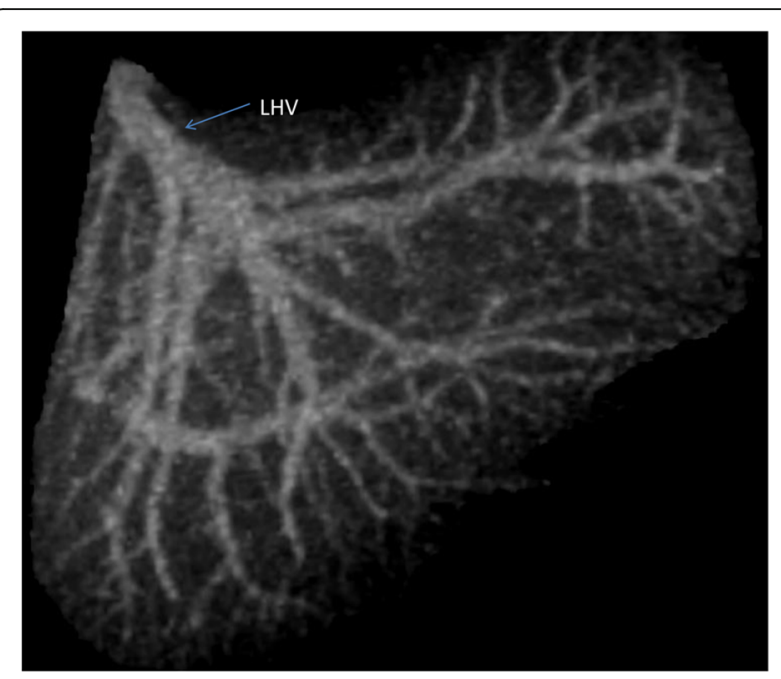

Fig. 9 Donor II Planned Left Lateral Sector Graft

was not established. In both grafts, no other back table reconstruction was needed.

\section{Sequential manner implantation of dual grafts Graft l- right lobe implantation and vascular anastomosis}

- The right lobe without MHV graft was orthotopically implanted on the right side of IVC.

- IVC was clamped using a side biting clamp. Cavotomy was performed at the orifice of RHV.

\section{- Hepatic venous anastomosis} Graft RHV was anastomosed to recipient IVC in an end to side manner with 5-0 polypropylene continuous suture.

Graft RIHV was anastomosed to recipient IVC in an end to side manner with 6-0 polypropylene continuous suture.

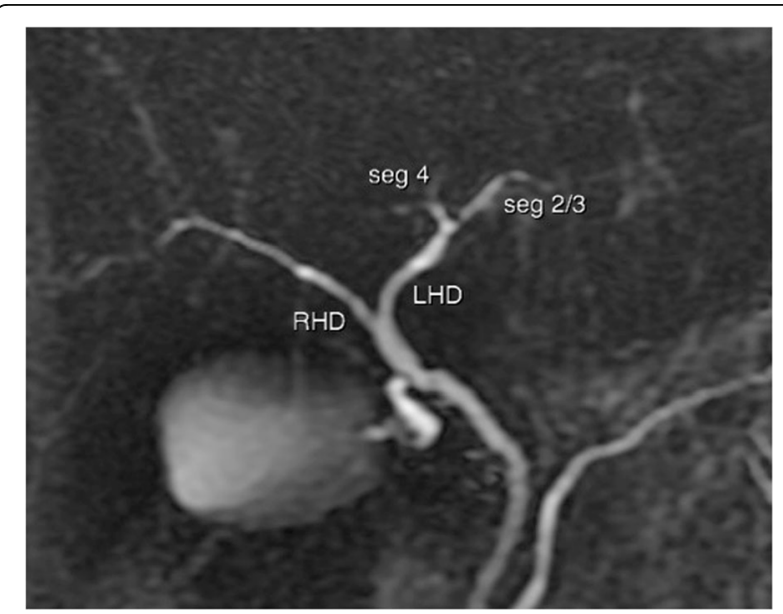

Fig. 10 Donor II Bile_Duct Anatomy (Huang Type A1B1) 
Table 3 Donor CT volumetric graft planning

\begin{tabular}{llll}
\hline Graft Type & Donor I & Donorll & Feasibility \\
\hline TLV & $941 c c$ & $986 c c$ & \\
Right Lobe with MHV & $589 c c$ & $592 c c$ & \\
Remnant & $352 c c$ & $394 c c$ & \\
GRWR & $(37 \%)$ & $(39.9 \%)$ & \\
Right Lobe without MHV & 0.65 & 0.65 & No \\
Remnant & $567 c c$ & $561 c c$ & \\
& $374 c c$ & 425 & \\
GRWR & $(39 \%)$ & $c c(43 \%)$ & \\
Left lobe & 0.63 & 0.62 & No \\
Remnant & $374 c c$ & $425 c c$ & \\
GRWR & 567 & 561 & \\
Left Lateral Lobe & $c c(60 \%)$ & $c c(57 \%)$ & \\
Remnant & 0.42 & 0.47 & No \\
GRWR & & $295 c c$ & \\
Dual Lobe = (Donor I) Right lobe & $567 c c$ & $295 c c$ & \\
without MHV + (Donorll) Left Lateral & & & No \\
Lobe & & $70 \%$ & \\
GRWR & & 0.32 & YES (0.95) \\
\hline
\end{tabular}

\section{- Portal Venous anastomosis}

Graft RPV was anastomosed to recipient RPV (end to end manner) using a 6-0 polypropylene continuous suture with appropriate growth factor. Graft reperfusion was immediate and uniform.

\section{- Arterial anastomosis:}

Graft RHA anastomosed with recipient RHA with 8-0 polypropylene interrupted suture under three time's magnification.

After arterial reperfusion intra-operative doppler ultrasound showed regular portal venous, hepatic venous and hepatic arterial flow.

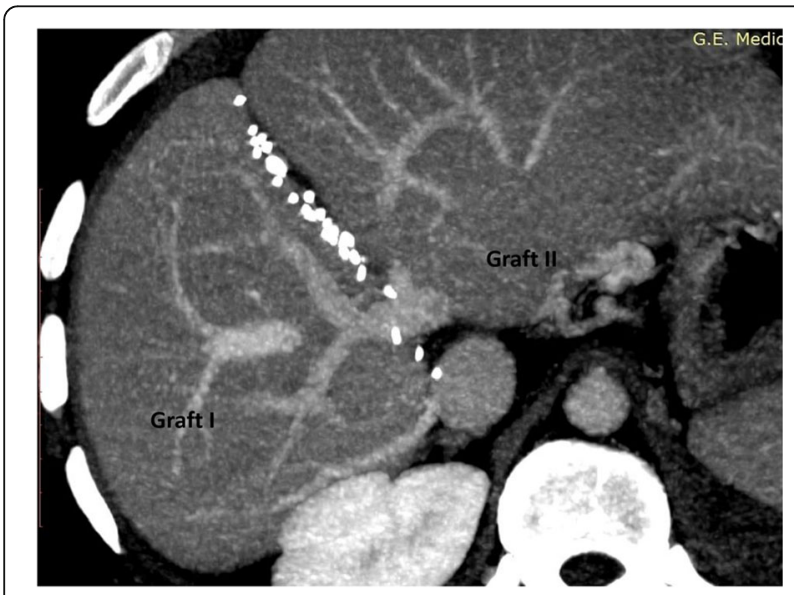

Fig. 11 Dual Graft I (Right lobe and Left latera I sector)
Table 4 Dual lobe -Actual graft weight and GRWR

\begin{tabular}{llll}
\hline Donor Type & Donor I & Donor II & Dual Lobe \\
\hline Graft Type & $\begin{array}{l}\text { Right Lobe } \\
\text { without MHV } \\
\text { Remnant }\end{array}$ & $\begin{array}{l}\text { Left } \\
\text { Lateral Lobe }\end{array}$ & $\begin{array}{l}\text { Right lobe without } \\
\text { MHV + Left Lateral } \\
\text { Lobe }\end{array}$ \\
$\begin{array}{l}\text { Actual graft } \\
\text { weight (Gm) }\end{array}$ & $503 \mathrm{gm}$ & $374 \mathrm{gm}$ & $877 \mathrm{gm}$ \\
GRWR & 0.55 & 0.41 & 0.96 \\
\hline
\end{tabular}

Graft II- left lateral lobe implantation and vascular anastomosis

\section{- Hepatic venous anastomosis}

Graft LHV was anastomosed to cavotomy made on recipient IVC at the orifice of LHV and MHV (end to side manner) with 5-0 polypropylene continuous suture.

- Portal Venous anastomosis

Graft LPV was anastomosed to recipient LPV (end to end manner) using a 6-0 polypropylene continuous suture

Graft reperfusion was immediate and uniform.

\section{- Arterial anastomosis:}

Graft LHA anastomosed with recipient segment IV hepatic artery with 8-0 polypropylene interrupted suture under three times magnification.

After arterial reperfusion intra-operative doppler ultrasound showed regular portal venous, hepatic venous and hepatic arterial flow in the left lateral graft as well.

\section{- Biliary anastomosis}

Number of graft ducts: two in right lobe graft (RASD and RPSD) and 1 in left lateral graft Number of anastomosis: 3

Anastomosis no 1: Graft RASD was anastomosed to the recipient right hepatic duct (RHD) with 6-0 Polydioxanone in an interrupted manner.

Anastomosis 2: Graft RPSD to the recipient cystic duct anastomosed with 6-0 Polydioxanone in an interrupted manner.

Anastomosis 3: Graft left hepatic duct (LHD) to the recipient LHD with 6-0 Polydioxanone in an interrupted manner. (Fig. 12).

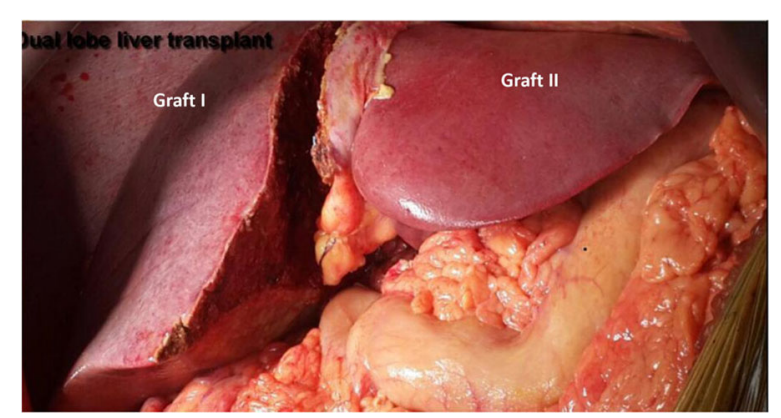

Fig. 12 Implanted Dual Grafts 


\section{Post-operative course \\ Recipient}

Recipient recovered well postoperatively. He received standard triple immunosuppressant. The patient was discharged on the eleventh postoperative day with normal liver graft function and good general condition.

\section{Donor}

Both donors recovered well postoperatively. They were discharged in good general condition and with normal liver function tests.

\section{Follow up}

The recipient was followed up weekly for the first 2 months and then every monthly. During follow up the patient was examined clinically and by laboratory tests including liver function tests, renal function tests, and serum tacrolimus level. A radiological assessment of hepatic vasculature was done whenever indicated. The long-term outcome, evaluated after a 48-month followup, was optimal with normal graft function, good general condition and superior quality of life.

\section{Discussion and conclusion}

There is an increasing incidence of ESLD and shortage of deceased donor organ pool especially in East Asian countries including India. LDLT has emerged as a crucial solution to tackle the situation of disparity between demand and supply of liver allografts. The evolution of LDLT is a history of search for an optimal graft to minimize donor risk and maximize recipient outcome simultaneously [6].

It has been shown that minimum GRWR of 0.8 is required to reduce the occurrence of SFSS [7]. Also most transplant surgeons accept a cut off of atleast 30\% remnant liver volume to optimize the donor's own safety. Undoubtedly, while planning LDLT, the safety of donors must have great importance and unavoidable risk for the donor must be balanced against the potential benefit for the recipient. For this reason, appropriate donors should provide a sufficient GRWR for the recipient while having a secure FLR after donation [8]. To overcome these problems, especially in large size recipients, dual lobe LDLT can be a feasible and realistic option [9].

\section{Ethical dilemma and drawbacks of dual lobe}

The surgical risk to the donor is always an ethical dilemma even with increasing application and success of LDLT. Donor death has an immense impact on the surviving recipient, other family members, and the transplant team. This ethical dilemma is compounded in dual lobe LDLT as two donors are at risk.

Left lobe donor hepatectomy is associated with lower morbidity and mortality than right lobe donor hepatectomy. Dual left lobe LDLT with two minor left lobe donor hepatectomies may actually have less combine donor risk than one major right lobe donor hepatectomy [10]. While donor mortality was estimated to be approximately 0.4 to $0.5 \%$ after right hepatectomy, risk of death for donors of a left lateral sector hepatectomy is $0.1 \%$ [11]. For this reason, the left lobe grafts (LL or LLS) are preferred over right lobe graft in dual lobe LDLT [12].

In the present case, it could have been possible to use reversed left lobe graft. However, we chose the Right lobe graft over the left lobe as we have maximum experience in Right Lobe LDLT. We have 100\% safety record for all our Right lobe donors (220 Cases). Moreover, reverse left dual graft surgery is technically challenging and associated with more complications.

The issue of donor risk can be partially defeated by expert dual lobe transplantation teams, technical advances and deliberately choosing the dual left lobe grafts or accepting the low GRWR single lobe graft with inflow modulation techniques like splenectomy, splenic artery ligation or porto-caval shunt. In our institute we prefer to use a porto-caval shunt if intra-operative actual GRWR is less than 0.7 [13]. Higher cost of the procedure is another drawback of the dual lobe LDLT, but this actually may be offset by the higher morbidity associated with SFSS, leading to cost escalation in SFS graft LDLT recipients.

In this case, there is a disparity between volumetric estimated graft weight (EGW- $295 \mathrm{~g}$ ) and actual graft weight (AGW-374 g) of the left lateral segment of donor II. Right and left lobes EGW on CT Volumetry is overestimated as compare to AGW. For the Left lateral segment (LLS) graft, CT may underestimate the volume because the actual surgical plane of transection is around $1 \mathrm{~cm}$ to the right of a falciform ligament whereas radiological plane is at falciform ligament. In addition to that non-hepatic tissues like falciform ligament which are excluded in CT volumetry are a part of LLS allograft included in AGW [14].

\section{Difficulties in surgical planning and decision making}

Surgical planning and decision-making process are complex and difficult in dual graft LDLT. Both donor and type of graft selection are very crucial for the optimal outcome. In our institute, we investigate donor as per protocol which includes laboratory investigations, estimation of liver attenuation index (LAI) on computed tomography, triphasic CT abdomen with the estimation of liver volumes. We asses biliary anatomy with MRCP preoperatively and with intra-operative cholangiogram.

Decision-making process of dual graft LDLT needs to be standardized and to achieve consensus across the transplant community, Yinzhe $\mathrm{Xu}$ et al. have proposed a 
decision making pathway for same [15] (Fig. 13). The implantation of two grafts requires huge technical precision like complex hepatic venous outflow reconstructions, tailored use of a cryopreserved interposition vein graft and anastomosis of multiple hepatic arteries, which are challenging and pivotal element of dual graft LDLT. Before implantation of the liver grafts, venoplasty of the hepatic veins in the grafts and the recipient should be performed to make wide outflow orifices and prevent outflow narrowing [16].

Post dual graft LDLT biliary complications incidence is around $21 \%$. Biliary reconstruction of both grafts can be performed using duct to duct anastomosis or combination of duct to duct and hepatico-jejunostomy $(\mathrm{HJ})$ to each graft. A biliary reconstruction strategy is therefore critical to the outcome of dual graft LDLT as well. Dual duct-to-duct anatomoses are preferred over $\mathrm{HJ}$ for being more physiologic and allowing better access for post-transplant endoscopic and interventional radiological procedures [17].

Furthermore, unnecessary dissection of recipient bile ducts in the hilar plate should be avoided and as much as connective tissue should be preserved to avoid ischemic biliary damage. Dual graft LDLT is an extremely

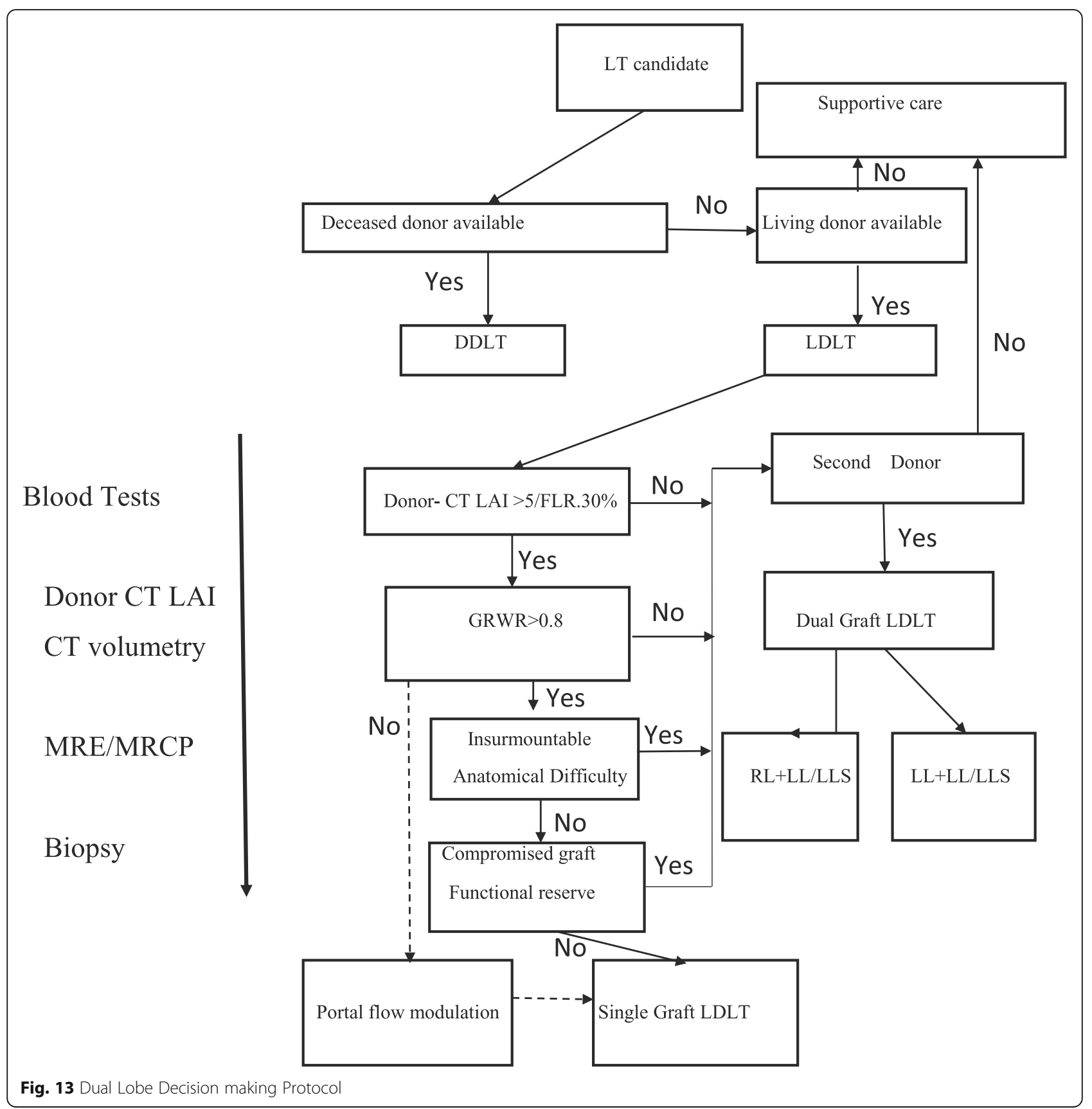


complex procedure, its outcome relies on the appropriate patient and donor selection, detailed donor evaluation, precise surgical planning, and meticulous surgical techniques [18].

In conclusion, the dual lobe LDLT is complex and technically challenging procedure and it should be considered a viable option to increase the donor pool and overcome the problem of the small-for-size syndrome.

\section{Acknowledgements}

The authors would like to thank Dr. Leena Tayshete and Mr. Rakesh Hire for language editing help and technical support. We have taken permission from them to use their full names in this section.

\section{Authors' contributions}

A) Substantial contributions to the conception or design of the work; or the acquisition, analysis, or interpretation of data for the work -VN, RM, AG, RB, PR, PG, AS, B) Drafting the work or revising it critically for important intellectual content- VN, RM, AG, RB, PR, PG, AS. C) Final approval of the version to be published- VN, RM, AG, RB, PR, PG, AS. D) Agreement to be accountable for all aspects of the work in ensuring that questions related tothe accuracy or integrity of any part of the work are appropriately investigated and resolved- VN, RM, AG, RB, PR, PG, AS.

\section{Funding}

No grant support or any kind of assistance from other institute.

\section{Availability of data and materials}

Not applicable.

\section{Ethics approval and consent to participate}

1. Institutional Ethics Committee, Global Hospital, Mumbai, India. 2. Recipient and both donors gave informed written consent for surgery and participation.

\section{Consent for publication}

Written and informed consent was taken from the recipient and both donors for the publication of this case report including consent for any potentially identifiable information and accompanying images. A copy of the written consent is available for review.

\section{Competing interests}

The authors declare that they have no competing interests.

Received: 13 February 2019 Accepted: 11 September 2019

Published online: 22 October 2019

\section{References}

1. Fan ST, Lo CM, Liu CL, Yong BH, Chan JK, Ng IO. Safety of donors in live donor liver transplantation using right lobe grafts. Arch Surg. 2000;135:336-40.

2. Kiuchi T, Kasahara M, Uryuhara K, Inomata Y, Uemoto S, Asonuma K, et al. Impact of graft size mismatching on graft prognosis in liver transplantation from living donors. Transplantation. 1999;67:321-7.

3. Yagi S, Uemoto S. Small-for-size syndrome in living donorliver transplantation. Hepatobiliary Pancreat Dis Int. 2012;11:570-6.

4. Soejima Y, Shimada M, Suehiro T, Hiroshige S, Niromiya M, Shiotani S, et al. Outcome analysis in adult-to-adult liver transplantation using the left lobe. Liver Transpl. 2003;9(6):581-6.

5. Lee S, Hwang S, Park K, Lee Y, Choi D, Ahn C, et al. An adult-to-adult living donor liver transplant using dual left lobe grafts. Surgery. 2001;129:647-50.

6. Lee SG, Hwang S, Park KM, Kim KH, Ahn CS, Lee YJ, et al. Seventeen adultto-adult living donor liver transplantations using dual grafts. Transplant Proc. 2001;33:3461-3.

7. Lo CM, Fan ST, Liu CL, Chan JK, Lam BK, Lau GK, et al. Minimum graft size for successful living donor liver transplantation. Transplantation. 1999;68: 1112-6.

8. Lee SG, Park KM, Hwang S, Lee YJ, Kim KH, Ahn CS, et al. Adult-to-adult living donor liver transplantation at the Asan medical center, Korea. Asian J Surg. 2002;25:277-84.
9. Moon D, Lee S, Hwang S, Kim K, Ahn C, Park K, et al. Resolution of severe graft steatosis following dual-graft living donor liver transplantation. Liver Transpl. 2006;12:1156-60.

10. Broering DC, Walter J, Rogiers $X$. The first two cases of living donor liver transplantation using dual grafts in Europe. Liver Transpl. 2007;13:149-53.

11. Otte JB. Donor complications and outcomes in live-liver transplantation. [comment]. Transplantation. 2003;75:1625-6.

12. Yang $\mathrm{CH}$, Chen $\mathrm{CL}$, Wang CC, Concejero AM, Wang SH, Liu YW, et al. Dual grafts in adult-to-adult living donor liver transplantation: a single center experience in Taiwan. Surgery. 2009;145:212-8.

13. Troisi R, Ricciardi S, Smeets P, Petrovic M, Van Maele G, Colle I, et al. Effects of hemi-portocaval shunts for inflow modulation on the outcome of smallfor-size grafts in living donor liver transplantation. Am J Transplant. 2005;5: 1397-404.

14. Goja S, Yadav SK, Yadav A, Piplani T, Rastogi A, Bhangui P, et al. Accuracy of preoperative $C T$ liver volumetry in living donor hepatectomy and its clinical implications. HepatoBiliary Surg Nutr. 2018;7(3):167-74.

15. Xu Y, Chen H, Yeh H, Wang H, Leng J, Dong J, et al. Living donor liver transplantation using DualGrafts: experience and lessons learned from cases worldwide. Liver Transpl. 2015;21:1438-48.

16. Lee SG. A complete treatment of adult living donor liver transplantation: a review of surgical technique and current challenges to expand indication of patients. Am J Transplant. 2015;15:17-38.

17. Hwang S, Lee SG, Lee YJ, Sung KB, Park KM, Kim KH, et al. Lessons learned from 1,000 living donor liver transplantations in a single center: how to make living donations safe. Liver Transpl. 2006:12:920-7.

18. Dong J, Yang S, Zeng J, Cai S, Ji W, Duan W, et al. Precision in liver surgery. Semin Liver Dis. 2013:33:189-203.

\section{Publisher's Note}

Springer Nature remains neutral with regard to jurisdictional claims in published maps and institutional affiliations.

Ready to submit your research? Choose BMC and benefit from:

- fast, convenient online submission

- thorough peer review by experienced researchers in your field

- rapid publication on acceptance

- support for research data, including large and complex data types

- gold Open Access which fosters wider collaboration and increased citations

- maximum visibility for your research: over $100 \mathrm{M}$ website views per year

At $\mathrm{BMC}$, research is always in progress.

Learn more biomedcentral.com/submissions 